\title{
Funções de distribuição diamétrica em um fragmento de Floresta Ombrófila Mista
}

\author{
Diametric distribution functions in a fragment of Mixed Ombrophylous Forest
}

\author{
Sebastião do Amaral MachadoI Alan Lessa Derci Augustynczik ${ }^{I I}$ \\ Rodrigo Geroni Mendes Nascimento ${ }^{\text {III }}$ Saulo Jorge Téo ${ }^{\text {II }}$ Eder Pereira MiguelI \\ Marco Aurélio Figura ${ }^{I I}$ Luís César Rodrigues da Silva ${ }^{\text {IV }}$
}

\section{RESUMO}

\begin{abstract}
Os objetivos deste estudo foram ajustar e selecionar funções de densidade probabilística que caracterizem a estrutura diamétrica desse fragmento e das espécies: Cedrela fissilis (Cedro), Luehea divaricata (Açoita Cavalo), Gochnatia polymorpha (Cambará), Sebastiania commersoniana (Branquilho) e Casearia sylvestris (Cafezeiro) de um fragmento de Floresta Ombrófila Mista com 15,24ha, localizado em Curitiba, Paraná. Para avaliar a distribuição diamétrica dessa floresta e das cinco espécies selecionadas, foram ajustadas sete funções probabilísticas para intervalos de classe de $5 \mathrm{~cm}$. Segundo os critérios de seleção adotados, o modelo que melhor representou a distribuição diamétrica para toda a floresta, o cedro, o cambará e o branquilho foi o de Weber; para o cafezeiro, a função Gamma (Adaptada) foi a mais eficiente $e$ para o açoita cavalo nenhuma dessas funções foi aderente. $O$ branquilho, o açoita cavalo, o cafezeiro e a floresta como um todo apresentaram distribuição em " J" invertido ou decrescente. Entretanto, o cedro e o cambará apresentaram distribuição tendendo à unimodalidade com forte assimetria à direita.
\end{abstract}

Palavras-chave: distribuição probabilística, teste de aderência, modelagem, comparação de funções.

\section{ABSTRACT}

The objective of this study was to adjust and select probability density functions that characterize the diametric structure of this forest fragment and for the species: Cedrela fissilis, Luehea divaricata, Gochnatia polymorpha, Sebastiania commersoniana and Casearia sylvestris for a 15.24 hectare fragment of Mixed Ombrophylous Forest, located in Curitiba, State of Paraná. In order to evaluate the diametric distributions of this forest and the five selected species, seven probability functions were adjusted for $5 \mathrm{~cm}$ class intervals. According to the criteria of selection adopted, the model that best represented the diametric distributions for the whole forest, Cedrela fissilis, Gochnatia polymorpha and Sebastiania commersoniana was the Weber model; for Casearia sylvestris, the Gamma function (adapted) was the most efficient, and for Luehea divaricata, none of these functions showed adherence. The Sebastiania commersoniana, Luehea divaricata, Casearia sylvestris and the forest as a whole showed inverted " $J$ " or decreasing distribution, while, Cedrela fissilis and Gochnatia polymorpha showed an almost unimodal distribution with a strong asymmetry to the right.

Key words: probability distribution, adherence test, modeling, function comparison.

\section{INTRODUÇÃO}

A Floresta Ombrófila Mista com araucária compreende as formações florestais típicas e exclusivas dos planaltos da região Sul do Brasil. Devido à intensa exploração dessas florestas ao longo dos anos por atividades como a exploração da madeira e, posteriormente, pelo avanço da agricultura e da pecuária, sua área passou de $73 \mathrm{mil} \mathrm{km}^{2}$ para aproximadamente 2.506.485 hectares no Estado do Paraná, distribuídos entre florestas em estágio inicial, médio e avançado de sucessão (SANQUETTA et al., 2001).

Para que as florestas com araucária sejam efetivamente conservadas e coloquem à disposição do homem todos os seus benefícios diretos e indiretos, é necessário gerar conhecimento acerca da estrutura e

'Departamento de Ciências Florestais, Universidade Federal do Paraná (UFPR). Av. Prefeito Lothário Meissner, 632, 80210-170,

Curitiba, PR, Brasil. E-mail: samachado@ufpr.br. Autor para correspondência.

"Programa de Pós-graduação em Engenharia Florestal, UFPR, Curitiba, PR, Brasil.

${ }^{\mathrm{II}}$ Curso de Engenharia Florestal, UFPR, Curitiba, PR, Brasil.

${ }^{\mathrm{IV}}$ Laboratório de Dendrometria, UFPR, Curitiba, PR, Brasil. 
da dinâmica dos remanescentes dessa formação. Nesse contexto, a distribuição diamétrica surge como um potente indicador da estrutura florestal, pois permite distinguir diferentes tipos florestais e fornece base para identificar a intensidade da regeneração natural para a espécie e para a floresta como um todo (SCOLFORO, 2006). Além disso, quando o uso da distribuição diamétrica é aliado a sucessivas medições da floresta, esta se torna uma poderosa ferramenta na avaliação da dinâmica de florestas nativas e plantadas. A distribuição diamétrica em florestas nativas heterogêneas e multiâneas ocorre sob a forma decrescente ou "J" invertido, indicando que a regeneração ocorre continuamente. Sabe-se que, por meio, da distribuição diamétrica pode-se identificar diferentes tipos florestais.

Atualmente, a melhor forma de descrever a estrutura diamétrica de uma floresta ou de uma espécie é por meio do emprego de funções de densidade probabilística (FDP). Essas funções vêm sendo utilizadas para a realização de diversas análises acerca da estrutura de variáveis das florestas, como, por exemplo: distribuição de frequências por classe de diâmetro para diferentes idades, sítios e densidades de povoamentos de Mimosa scabrella (BARTOSZECK et al., 2004), prognose do volume por classe diamétrica para povoamentos de Eucalyptus grandis (ABREU et al., 2002), dentre outras aplicações. Conhecer a distribuição diamétrica, principalmente de florestas naturais, é de suma importância para o seu manejo sustentado, e as funções de densidade probabilística têm sido uma importante ferramenta para esse fim.

A Floresta Ombrófila Mista é uma das tipologias vegetais da região Sul do Brasil com maior riqueza e diversidade de espécies. No entanto, algumas dessas espécies são particularmente importantes nessa formação florestal por apresentarem um elevado valor de importância, como é o caso do açoita cavalo, cedro, branquilho, cambará e cafezeiro.

Os objetivos do trabalho foram identificar o tipo de distribuição diamétrica, ajustar e selecionar funções de densidade probabilística para a distribuição diamétrica para todo o fragmento de Floresta Ombrófila Mista, bem como para algumas espécies desse fragmento.

\section{MATERIAL E MÉTODOS}

O estudo foi conduzido em um fragmento de Floresta Ombrófila Mista, conhecido como Capão da Engenharia Florestal, situado em Curitiba, Paraná (PR), entre as coordenadas $25^{\circ} 26^{\prime} 50^{\prime \prime}$ e $25^{\circ} 27^{\prime} 33^{\prime \prime} \mathrm{S}$ e 49 14 ' $16^{\prime \prime}$ e $49^{\circ} 14^{\prime} 33^{\prime \prime} \mathrm{W}$. Esse remanescente ocupa uma área total de 15,24ha, dos quais 12,96ha são ocupados por Floresta Ombrófila Mista. Em algumas partes, podese observar um nível de antropização bastante acentuado, sobretudo em sua bordadura. Nessas partes, prevalecem capoeiras com presença marcante de taquarais, com cerca de 2,28ha. O clima predominante, segundo Koppën, é do tipo Cfb: subtropical úmido mesotérmico. RONDON NETO et al. (2002) identificaram nessa área a existência de Solos Hidromórficos próximos aos canais de drenagem e de Cambissolos Podzólicos nas regiões mais drenadas.

Os dados utilizados provieram do censo realizado no Capão da Engenharia Florestal durante os anos de 2006 e 2007 pelo Laboratório de Dendrometria (LADEN) da Universidade Federal do Paraná. Ao todo, 9.837 árvores, pertencentes a 118 espécies, compõem o banco de dados do censo do Capão da Engenharia Florestal. Porém, para a modelagem da distribuição diamétrica da floresta como um todo, foram utilizados dados de 9.502 árvores, pois a araucária foi excluída da análise por se tratar de uma espécie com comportamento diferenciado, a qual está sendo estudada separadamente.

Para a modelagem da distribuição diamétrica de Cedrela fissilis (cedro), Luehea divaricata (açoita cavalo), Gochnatia polymorpha (cambará), Sebastiania commersoniana (branquilho) e Casearia sylvestris (cafezeiro) foram utilizados, respectivamente, dados de 204, 762, 276, 163 e 1.390 árvores. Para cada espécie estudada, assim como para a floresta, foram ajustadas e testadas sete funções de densidade probabilística (Tabela 1), utilizando intervalos de classe de $5 \mathrm{~cm}$, para a obtenção das frequências por classe de diâmetro.

Para avaliar a estrutura diamétrica da floresta, foi necessário realizar um levantamento quantitativo dos elementos que a compõe. Nesse sentido, foram computadas algumas estatísticas descritivas para serem obtidas informações preliminares das características dessa estrutura. Essas informações foram geradas a partir da variável diâmetro à altura do peito (DAP), para os dados da floresta e para as cinco espécies selecionadas.

Para determinar os parâmetros das funções de densidade de probabilidade e posterior estimativa do número de árvores, foi utilizada a planilha do programa MS EXCEL 2007. O software possibilitou estimar os parâmetros das distribuições Weibull 2P e 3P, Weber, Exponencial e Gamma (adaptada), por meio da interação de valores iniciais paramétricos, "sementes", pelo método de mínimos quadrados, por meio do algoritmo de Levenberg Marquardt. Os coeficientes das funções SB de Johnson e Gamma foram determinados por meio do emprego do Método dos Momentos.

A função Gamma, em sua forma original, apresenta os coeficientes de seu denominador vinculados ao numerador. Dessa forma, a função pode se tornar mais rígida comparativamente a um modelo cujo denominador não está atrelado ao seu numerador. Sendo assim, para flexibilizar o modelo, partiu-se do 
Tabela 1 - Funções de densidade probabilística testadas e suas condições de existência.

\begin{tabular}{|c|c|c|}
\hline Distribuição & Função de Densidade Probabilística & Condições \\
\hline Gamma & $f(x)=\frac{\left(x-x_{\min }\right)^{\alpha-1} e^{-\left(\frac{1}{\beta}\right) \cdot\left(x-x_{\min }\right)}}{\Gamma(\alpha) \beta^{\alpha}}$ & $\begin{array}{l}x \geq x_{\min } \\
\alpha, \beta>0 \\
-\infty<x_{\min }<x_{\max }<+\infty\end{array}$ \\
\hline $\begin{array}{l}\text { Gamma } \\
\text { (Adaptada) }\end{array}$ & $f(x)=\frac{\left(x-x_{\min }\right)^{\alpha-1} e^{-\left(\frac{1}{\beta}\right) \cdot\left(x-x_{\min }\right)}}{\gamma}$ & $\begin{array}{l}x \geq x_{\min } \\
\alpha, \beta, \gamma>0 \\
-\infty<x_{\min }<x_{\max }<+\infty\end{array}$ \\
\hline Weibull 2P & $f(x)=\left(\frac{c}{b}\right)\left(\frac{x}{b}\right)^{c-1} e^{\left[-\left(\frac{x}{b}\right)^{c}\right]}$ & $\begin{array}{l}x \geq 0 \\
b, c>0\end{array}$ \\
\hline Weibull 3P & $f(x)=\left(\frac{c}{b}\right)\left(\frac{x-a}{b}\right)^{c-1} e^{\left[-\left(\frac{x-a}{b}\right)^{c}\right]}$ & $\begin{array}{l}a \leq x<\infty \\
a \geq 0 \\
b, c>0\end{array}$ \\
\hline Sb de Johnson & $f(x)=\frac{\delta}{\sqrt{2 \pi}} \frac{\lambda}{(d-\varepsilon)(\lambda+\varepsilon-d)} e^{\left\{-\frac{1}{2}\left[\gamma+\delta \ln \left(\frac{\delta-\varepsilon}{\lambda+\varepsilon-d}\right)\right]^{2}\right\}}$ & $\begin{array}{l}\varepsilon<d<\varepsilon+\lambda \\
-\infty<\varepsilon<+\infty \\
\lambda>0 \\
-\infty<\gamma<\infty \\
\delta>0\end{array}$ \\
\hline Exponencial & $f(x)=\lambda \cdot e^{-x \cdot \lambda}$ & $x \geq 0$ \\
\hline Weber & $f(x)=\frac{x^{a}}{(b+c . x)^{d}}$ & $\begin{array}{l}0 \leq x<+\infty \\
d \geq a+1 \\
d \neq a+2 \\
d \neq a+3 \\
a, b, c>0\end{array}$ \\
\hline
\end{tabular}

Legenda: $f(x)=$ Função densidade de probabilidade da variável $x ; x=$ Variável aleatória; $x_{\min }=$ Valor mínimo de $x ; \pi=$ Constante PI $(3,1416 \ldots) ; e=$ Constante de Euler $(2,7182 \ldots) ; a, b, c, d, \alpha, \beta, \delta, \varepsilon, \gamma, \lambda=$ Parâmetros a serem estimados.

pressuposto de que, ao substituir o denominador original por outro que não apresentasse o coeficiente vinculado ao numerador, seria possível obter melhores ajustes para esse modelo, além de torná-lo mais simples. A função Gamma (adaptada) é apresentada na tabela 1. Embora tenham sido estimados alguns valores estatísticos para os modelos testados, tais como: coeficiente de determinação ajustado $\left(R^{2} a j\right)$ e erro padrão da estimativa em porcentagem (Syx\%), o critério final utilizado na seleção da função para descrever a distribuição diamétrica da floresta e das cinco espécies selecionadas foi o teste de KolmogorovSmirnov, adotando 5\% de probabilidade de erro. Além dessas análises, também foram traçadas curvas das frequências estimadas sobre o histograma das frequências observadas.

\section{RESULTADOS E DISCUSSÃO}

Foi possível verificar que as espécies que apresentam menor dispersão em relação à média dos DAP's foram o branquilho e o cafezeiro (Tabela 2). A 
Tabela 2 - Estatísticas descritivas dos conjuntos de dados da variável DAP.

\begin{tabular}{|c|c|c|c|c|c|c|}
\hline \multirow{2}{*}{ Medida } & \multirow{2}{*}{ Floresta } & \multicolumn{5}{|c|}{ - } \\
\hline & & Cedro & Açoita Cavalo & Cambará & Branquilho & Cafezeiro \\
\hline Frequência & 9502 & 204 & 762 & 276 & 163 & 1390 \\
\hline Média & 16,8 & 23,9 & 18,6 & 20,8 & 15,2 & 13,7 \\
\hline Mediana & 14,4 & 21,1 & 14,3 & 19,4 & 14,4 & 12,9 \\
\hline Moda & 10,0 & 11,3 & Múltipla & Múltipla & 11,8 & Múltipla \\
\hline DAPmin & 10,0 & 10,0 & 10,0 & 10,0 & 10,0 & 10,0 \\
\hline Amplitude & 68,9 & 59,4 & 68,4 & 35,6 & 25,0 & 36,5 \\
\hline Q1 & 11,9 & 16,1 & 12,1 & 15,0 & 11,9 & 11,4 \\
\hline Q3 & 18,9 & 27,9 & 18,5 & 24,9 & 17,7 & 15,1 \\
\hline Interquartil & 7,0 & 11,8 & 6,4 & 9,8 & 5,8 & 3,7 \\
\hline $\mathrm{S}^{2}$ & 57,1 & 133,5 & 146,4 & 54,6 & 17,2 & 10,6 \\
\hline $\mathrm{S}$ & 7,6 & 11,6 & 12,1 & 7,4 & 4,2 & 3,3 \\
\hline $\mathrm{CV}$ & $45 \%$ & $48 \%$ & $65 \%$ & $36 \%$ & $27 \%$ & $24 \%$ \\
\hline Assimetria & 2,6 & 1,7 & 2,5 & 1,0 & 1,2 & 2,4 \\
\hline Curtose & 9,6 & 3,5 & 5,9 & 0,7 & 2,3 & 12,7 \\
\hline
\end{tabular}

análise da medida de dispersão interquartil $\left(\mathrm{Q}_{3}-\mathrm{Q}_{1}\right)$ revelou que $50 \%$ dos dados estão compreendidos em uma amplitude de 7,0;11,8; 6,4; 9,8; 5,8 e 3,7 cm de DAP, respectivamente para a floresta, o cedro, o açoita cavalo, o cambará, o branquilho e o cafezeiro, demonstrando que há um elevado grau de agrupamento dos dados.

Para avaliação da concentração de árvores por classe de diâmetro, tomou-se como base a tabela 3 , que apresentada as frequências relativas por classe diamétrica em relação à frequência observada. Foi possível observar que, para a floresta e para o açoita cavalo, cerca de $55 \%$ dos indivíduos estão concentrados na classe de diâmetro que vai de 10 a $15 \mathrm{~cm}$. Para o cedro, a maior concentração $(64,7 \%)$ ocorre nas três primeiras classes, compreendidas entre os diâmetros de 10 a $25 \mathrm{~cm}$. Aproximadamente $53 \%$ dos indivíduos de cambará possuem DAP's variando entre 10 e $20 \mathrm{~cm}$. Cerca de $87 \%$ dos indivíduos de branquilho e $96 \%$ das árvores de cafezeiro ocupam as duas primeiras classes de diâmetro, cuja variação é de 10 a $20 \mathrm{~cm}$. Com isso, conclui-se que o espaço é ocupado por indivíduos de pequenas dimensões.

Estudando uma Floresta Ombrófila Mista, localizada em São João do Triunfo - PR, SCHAAF et al. (2006) encontraram maior número de indivíduos de grande porte comparativamente ao encontrado no presente estudo. No caso do Capão da Engenharia Florestal, observa-se que a floresta apresentou apenas

Tabela 3 - Frequência relativa por classe diamétrica.

\begin{tabular}{|c|c|c|c|c|c|c|}
\hline \multirow{2}{*}{ Centro de Classe } & \multicolumn{6}{|c|}{ 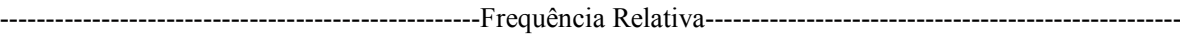 } \\
\hline & Floresta & Cedro & Açoita Cavalo & Cambará & Branquilho & Cafezeiro \\
\hline 12,5 & $55,0 \%$ & $19,6 \%$ & $55,8 \%$ & $24,6 \%$ & $57,1 \%$ & $74,2 \%$ \\
\hline 17,5 & $23,7 \%$ & $26,0 \%$ & $23,8 \%$ & $28,3 \%$ & $30,1 \%$ & $21,9 \%$ \\
\hline 22,5 & $10,2 \%$ & $19,1 \%$ & $5,9 \%$ & $22,5 \%$ & $11,0 \%$ & $3,0 \%$ \\
\hline 27,5 & $4,8 \%$ & $12,8 \%$ & $2,4 \%$ & $12,0 \%$ & $1,2 \%$ & $0,5 \%$ \\
\hline 32,5 & $3,0 \%$ & $9,3 \%$ & $1,7 \%$ & $7,6 \%$ & $0,0 \%$ & $0,2 \%$ \\
\hline 37,5 & $1,5 \%$ & $5,4 \%$ & $2,4 \%$ & $2,5 \%$ & $0,6 \%$ & $0,1 \%$ \\
\hline 42,5 & $0,7 \%$ & $2,0 \%$ & $1,4 \%$ & $1,8 \%$ & - & $0,0 \%$ \\
\hline 47,5 & $0,5 \%$ & $2,0 \%$ & $1,6 \%$ & $0,7 \%$ & - & $0,1 \%$ \\
\hline 52,5 & $0,3 \%$ & $1,0 \%$ & $1,6 \%$ & - & - & - \\
\hline 57,5 & $0,2 \%$ & $1,0 \%$ & $1,7 \%$ & - & - & - \\
\hline 62,5 & $0,1 \%$ & $0,0 \%$ & $0,7 \%$ & - & - & - \\
\hline 67,5 & $0,1 \%$ & $2,0 \%$ & $0,7 \%$ & - & - & - \\
\hline 72,5 & $0,0 \%$ & - & $0,3 \%$ & - & - & - \\
\hline 77,5 & $0,0 \%$ & - & $0,3 \%$ & - & - & - \\
\hline Total & $100 \%$ & $100 \%$ & $100 \%$ & $100 \%$ & $100 \%$ & $100 \%$ \\
\hline
\end{tabular}

Ciência Rural, v.39, n.8, nov, 2009. 
0,72\% dos seus indivíduos com mais de $50 \mathrm{~cm}$ de DAP, enquanto que a área de São João do Triunfo apresentou um total de $8,99 \%$ no levantamento realizado no ano 2000 .

Verificou-se que apenas cedro e açoita cavalo atingem as classes de diâmetro superiores, enquanto as demais espécies não ultrapassam os $50 \mathrm{~cm}$ de DAP. Esse fato também foi observado por SCHAAF et al. (2006), analisando um outro grupo de espécies da Floresta Ombrófila Mista. Esses autores atribuíram isso a duas características intrínsecas a cada espécie - seu tamanho limite e sua longevidade - e uma característica ecológica - a competição.

Ajuste das funções de densidade probabilística

$\mathrm{Na}$ tabela 4, são apresentados os parâmetros obtidos no ajuste dos modelos de distribuição diamétrica. Esses valores foram utilizados diretamente nas funções probabilísticas para obtenção da frequência por classe diamétrica da floresta, do cedro, do açoita cavalo, do cambará, do branquilho e do cafezeiro.
Como pode ser visualizado na tabela 5 , os valores $D_{\text {cal }}$ do teste de Kolmogorov-Smirnov que foram menores que o tabelado indicam a não rejeição da hipótese de nulidade, concluindo-se que as frequências esperadas e observadas são similares sob ponto de vista estatístico. Em contrapartida, os ajustes que apresentaram valores significativos são inadequados para descrever o conjunto de dados. Analisando-se a tabela 5, foi possível verificar que a função que apresentou os menores erros de estimativa e os maiores valores do coeficiente de determinação ajustado foi a de Weber. Também foi possível verificar que o modelo Gamma (Adaptado) propiciou um desempenho melhor que o modelo original.

Pelas análises estatísticas empregadas, foi possível concluir que a função proposta por Weber apresentou maior eficiência para representar a distribuição diamétrica da floresta, do cedro, do cambará e do branquilho, já para o cafezeiro a função que melhor representou sua série de diâmetros foi a função Gamma (Adaptada), enquanto que para o açoita cavalo nenhuma função foi aderente.

Tabela 4 - Parâmetros estimados das funções de distribuição diamétrica.

\begin{tabular}{|c|c|c|c|c|c|c|c|}
\hline \multirow{2}{*}{ Função } & \multirow{2}{*}{ Coef. } & \multirow{2}{*}{ Floresta } & \multicolumn{5}{|c|}{---Espécies---------------------------------------' } \\
\hline & & & Cedro & Açoita Cavalo & Cambará & Branquilho & Cafezeiro \\
\hline \multirow{3}{*}{ Weibull 3P } & a & 10,224 & 8,754 & 8,741 & 9,516 & 9,201 & 9,465 \\
\hline & $\mathrm{b}$ & 6,088 & 15,539 & 5,876 & 12,687 & 6,595 & 4,661 \\
\hline & $\mathrm{c}$ & 0,939 & 1,522 & 1,311 & 1,544 & 1,404 & 1,429 \\
\hline \multirow{2}{*}{ Weibull 2P } & $\mathrm{b}$ & 13,970 & 21,980 & 13,979 & 20,394 & 14,551 & 14,321 \\
\hline & $\mathrm{c}$ & 3,952 & 2,441 & 4,099 & 2,765 & 4,562 & 6,320 \\
\hline \multirow{2}{*}{ Gamma } & $\alpha$ & 0,905 & 1,509 & 1,638 & 2,059 & 1,768 & 1,728 \\
\hline & $\beta$ & 7,808 & 9,271 & 3,186 & 5,282 & 3,063 & 2,333 \\
\hline \multirow{3}{*}{ Gamma (Adaptada) } & $\alpha$ & 0,874 & 1,833 & 1,638 & 1,893 & 1,826 & 2,259 \\
\hline & $\beta$ & 6,990 & 7,401 & 3,185 & 6,235 & 3,236 & 1,915 \\
\hline & $\gamma$ & 5,691 & 38,409 & 7,350 & 30,986 & 8,624 & 5,792 \\
\hline \multirow{4}{*}{ Weber } & a & 3,195 & 10,294 & 7,886 & 6,708 & 6,356 & 14,319 \\
\hline & $\mathrm{b}$ & 1,309 & 2,364 & 1,385 & 1,530 & 1,105 & 1,331 \\
\hline & $\mathrm{c}$ & 0,121 & 0,140 & 0,040 & 0,024 & 0,006 & 0,030 \\
\hline & $\mathrm{d}$ & 9,909 & 20,659 & 34,844 & 33,034 & 109,833 & 70,924 \\
\hline \multirow{4}{*}{ Sb de Johnson } & $\delta$ & 0,966 & 0,907 & 0,729 & 0,990 & 1,114 & 1,277 \\
\hline & $\lambda$ & 78,88 & 69,39 & 78,46 & 45,65 & 35,05 & 46,51 \\
\hline & $\varepsilon$ & 10,03 & 10,03 & 10,03 & 10,03 & 10,03 & 10,03 \\
\hline & $\gamma$ & 2,281 & 1,321 & 1,543 & 1,229 & 1,956 & 3,046 \\
\hline Exponencial & $\lambda$ & 0,074 & 0,052 & 0,076 & 0,056 & 0,0750 & 0,0814 \\
\hline
\end{tabular}


Tabela 5 - Teste de aderência de Kolmogorov-Smirnov, erro padrão da estimativa (Syx\%) e coeficiente de determinação ajustado ( ${ }^{2}{ }_{\text {aj) }}$ ), para as funções testatadas.

\begin{tabular}{|c|c|c|c|c|c|c|c|}
\hline \multirow[b]{2}{*}{ Distribuição } & \multirow[b]{2}{*}{ Estatística } & \multirow[b]{2}{*}{ Floresta } & \multicolumn{5}{|c|}{ 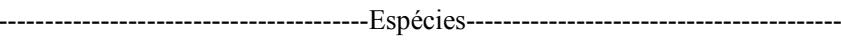 } \\
\hline & & & Cedro & Açoita Cavalo & Cambará & Branquilho & Cafezeiro \\
\hline \multirow{4}{*}{ Weibull 3P } & $\mathrm{D}_{\text {tab }}(5 \%)$ & 0,014 & 0,095 & 0,049 & 0,082 & 0,107 & 0,036 \\
\hline & $\mathrm{D}_{\text {calc }}$ & 0,011 & 0,021 & $0,121 *$ & 0,017 & 0,039 & 0,005 \\
\hline & Syx \% & $0,14 \%$ & $3,19 \%$ & $2,26 \%$ & $0,95 \%$ & $1,33 \%$ & $0,07 \%$ \\
\hline & $\mathrm{R}^{2} \mathrm{aj}$ & 1,000 & 0,984 & 0,993 & 0,996 & 0,997 & 1,000 \\
\hline \multirow{4}{*}{ Weibull 2P } & $\mathrm{D}_{\text {tab }}(5 \%)$ & 0,014 & 0,095 & 0,049 & 0,082 & 0,107 & 0,036 \\
\hline & $\mathrm{D}_{\text {calc }}$ & $0,216^{*}$ & $0,132 *$ & $0,205^{*}$ & $0,125^{*}$ & 0,021 & $0,115^{*}$ \\
\hline & Syx \% & $1,60 \%$ & $4,85 \%$ & $3,73 \%$ & $2,53 \%$ & $5,10 \%$ & $1,33 \%$ \\
\hline & $R^{2} a j$ & 0,959 & 0,962 & 0,982 & 0,968 & 0,973 & 0,992 \\
\hline \multirow{4}{*}{ Gamma } & $\mathrm{D}_{\mathrm{tab}}(5 \%)$ & 0,014 & 0,095 & 0,049 & 0,082 & 0,107 & 0,036 \\
\hline & $\mathrm{D}_{\text {calc }}$ & $0,066^{*}$ & 0,042 & $0,251 *$ & $0,092 *$ & $0,120 *$ & $0,117^{*}$ \\
\hline & Syx \% & $0,92 \%$ & $4,37 \%$ & $10,33 \%$ & $3,13 \%$ & $5,32 \%$ & $2,28 \%$ \\
\hline & $\mathrm{R}^{2} \mathrm{aj}$ & 0,986 & 0,970 & 0,863 & 0,952 & 0,951 & 0,976 \\
\hline \multirow{4}{*}{ Gamma (Adaptada) } & $\mathrm{D}_{\mathrm{tab}}(5 \%)$ & 0,014 & 0,095 & 0,049 & 0,082 & 0,107 & 0,036 \\
\hline & $\mathrm{D}_{\text {calc }}$ & 0,012 & 0,018 & $0,116^{*}$ & 0,010 & 0,009 & 0,004 \\
\hline & Syx \% & $0,15 \%$ & $1,20 \%$ & $2,20 \%$ & $1,23 \%$ & $1,02 \%$ & $0,06 \%$ \\
\hline & $\mathrm{R}^{2} \mathrm{aj}$ & 1,000 & 0,995 & 0,994 & 0,993 & 0,998 & 1,000 \\
\hline \multirow{4}{*}{ Weber } & $\mathrm{D}_{\mathrm{tab}}(5 \%)$ & 0,014 & 0,095 & 0,049 & 0,082 & 0,107 & 0,036 \\
\hline & $\mathrm{D}_{\text {calc }}$ & 0,004 & 0,015 & $0,113^{*}$ & 0,003 & 0,012 & 0,005 \\
\hline & Syx \% & $0,10 \%$ & $2,42 \%$ & $2,21 \%$ & $0,88 \%$ & $0,88 \%$ & $0,06 \%$ \\
\hline & $\mathrm{R}^{2} \mathrm{aj}$ & 1,000 & 0,991 & 0,994 & 0,996 & 0,999 & 1,000 \\
\hline \multirow{4}{*}{ Sb de Johnson } & $\mathrm{D}_{\text {tab }}(5 \%)$ & 0,014 & 0,095 & 0,049 & 0,082 & 0,107 & 0,036 \\
\hline & $\mathrm{D}_{\text {calc }}$ & $0,090^{*}$ & 0,056 & $0,196^{*}$ & 0,036 & $0,142 *$ & $0,230^{*}$ \\
\hline & Syx \% & $1,58 \%$ & $4,97 \%$ & $10,47 \%$ & $1,79 \%$ & $3,71 \%$ & $3,41 \%$ \\
\hline & $\mathrm{R}^{2} \mathrm{aj}$ & 0,960 & 0,961 & 0,859 & 0,984 & 0,976 & 0,947 \\
\hline \multirow{4}{*}{ Exponencial } & $\mathrm{D}_{\text {tab }}(5 \%)$ & 0,014 & 0,095 & 0,049 & 0,082 & 0,107 & 0,036 \\
\hline & $\mathrm{D}_{\text {calc }}$ & $0,571 *$ & $0,453 *$ & $0,548^{*}$ & $0,520^{*}$ & $0,664^{*}$ & $0,716^{*}$ \\
\hline & Syx \% & $6,13 \%$ & $18,24 \%$ & $22,04 \%$ & $12,66 \%$ & $22,29 \%$ & $13,10 \%$ \\
\hline & $\mathrm{R}^{2} \mathrm{aj}$ & 0,394 & 0,469 & 0,375 & 0,209 & 0,145 & 0,215 \\
\hline
\end{tabular}

*Significativo a $5 \%$ de probabilidade de erro no teste bilateral.

O melhor desempenho do modelo de Weber era esperado, já que essa função apresenta maior flexibilidade em relação aos demais modelos testados. Esse mesmo autor, ao modelar a distribuição de alturas da regeneração natural de Ocotea porosa, concluiu que os modelos clássicos não resultaram em ajustes satisfatórios, e o único que se ajustou satisfatoriamente foi o modelo proposto por ele, chamado neste trabalho de Weber. Mesmo apresentando um número menor de parâmetros, o desempenho da função Weibull de três parâmetros foi similar ao da função de Weber.

O modelo de Weber ajustado para a floresta e para o cambará apresentou distribuição de resíduos satisfatória, com baixos erros por classe de diâmetro. Os demais modelos, apesar de apresentarem uma boa estimativa total do número de árvores, mostraram tendência em subestimar o número de árvores nas classes superiores, em que se encontram os indivíduos de maior porte.

Como previsto na análise preliminar dos dados, as curvas de distribuição diamétrica apresentaram grande concentração de indivíduos nas classes de DAP inferiores, e esta se apresenta sob a forma de "J invertido" para a floresta, o açoita cavalo, o branquilho e o cafezeiro. SCHAAF et al. (2006), em estudo realizado numa Floresta Ombrófila Mista, localizada em São João do Triunfo - PR, também observaram que a distribuição diamétrica da floresta ocorreu sob a forma de "J invertido".

A distribuição de diâmetros do cedro e do cambará apresentaram-se sob a forma unimodal, porém com forte assimetria à direita. Esse fato vai de encontro 
com as observações feitas por SCOLFORO (2006), o qual afirmou que, em nível de espécie, é possível obter distribuições de diâmetros que se assemelhem à distribuição normal com alguma assimetria.

\section{CONCLUSÕES}

A floresta como um todo, bem como o açoita cavalo, o branquilho e o cafezeiro, apresentaram distribuição exponencial negativa, já o cambará e o cedro apresentaram distribuição unimodal com assimetria positiva.

O modelo de Weber foi o que apresentou melhor ajuste para a distribuição diamétrica de todo o fragmento, para o cedro, para o cambará e para o branquilho, enquanto a distribuição Gamma (Adaptada) foi a que melhor se ajustou à distribuição de diâmetros do cafezeiro.

\section{REFERÊNCIAS}

ABREU, E.C.R. et al. Modelagem para prognose precoce do volume por classe diamétrica para Eucalyptus grandis. Scientia Forestalis, Piracicaba, n.61, p.86-102, 2002.
BARTOSZECK, A.P.S. et al. Distribuição diamétrica para bracatingais em diferentes idades, sítios e densidades da região metropolitana de Curitiba. Revista Floresta, Curitiba, v.34, n.3, p.305-324, 2004.

RONDON NETO, R.M. et al. Caracterização florística e estrutural de um fragmento de floresta ombrófila mista, em Curitiba, PR - Brasil. Revista Floresta, Curitiba, v.32, n.1, p.3-16, 2002.

SANQUETTA, C.R. et al. Dinâmica da estrutura horizontal de um fragmento de Floresta Ombrófila Mista no Centro-Sul do Paraná. Revista de Ciências Exatas e Naturais, Irati, v.3, n. 1, p.43-47, 2001.

SCHAAF, L.B. et al. Alteração na estrutura diamétrica de uma floresta ombrófila mista no período entre 1979 e 2000 . Revista Árvore, Viçosa, v.30, n.2, p.283-295, 2006. Disponível em < http://www.scielo.br/scielo.php?script=sci_arttext\&pid=S0100$67622006000200016 \& \operatorname{lng}=\mathrm{en} \& \mathrm{nrm}=$ iso $>$. Acesso em: $10 \mathrm{jul}$. 2009. doi: 10.1590/S0100-67622006000200016.

SCOLFORO, J.R.S. Biometria florestal: modelos de crescimento e produção florestal. Lavras: UFLA/FAEPE, 2006. 393p. 